\title{
Analysis of Cannabinoids in Hemp Plants
}

\author{
Christian Giroud*
}

\begin{abstract}
In 2001, guidelines concerning cannabis were issued by the Group of Forensic Chemistry of the Swiss Society of Forensic Medicine. These guidelines deal with the sampling of Cannabis plants and their processing before analysis. Some recommendations are also suggested for the determination of $\Delta^{9}$-tetrahydrocannabinol $\left(\Delta^{9}-\mathrm{THC}\right)$. In this paper, the biosynthetic pathway of $\Delta^{9}-\mathrm{THC}$ is presented and the putative ecological function of the cannabinoids discussed. The taxonomy and botany of the hemp plant are also considered. The influence of age, sex, and plant part on the accumulation of $\Delta^{9}$-THC is discussed. The need for criteria aimed at the distinction between fiber hemp and cannabis belonging to the drug type is especially emphasized. The current Swiss situation and legislation are briefly presented. Furthermore, the sampling of cannabis material, its processing and analysis are described, alternative strategies for the characterization of hemp exhibits are also presented.
\end{abstract}

Keywords: Cannabinoids · Cannabis · Forensics · Hemp analysis · Profiling of cannabis products · THC

\section{Introduction}

Cannabis is the most commonly used illicit drug in Switzerland. Thorough clinical studies have demonstrated that among more than 70 different cannabinoids, $\Delta^{9}$-tetrahydrocannabinol $\left(\Delta^{9}\right.$-THC) is the main active compound responsible for the psychological and physiological effects of cannabis in man [1]. Only $\Delta^{8}$-tetrahydrocannabinol, which is present in very low concentrations in the plant, but can be obtained quite easily by chemical synthesis or formed as an artifact from $\Delta^{9}$-THC [2], has shown similar psychoactive properties. Cannabinol, which is essentially a chemical degradation product of $\Delta^{9}$-THC, has also shown some activity; its concentration generally increases as samples age. Fresh specimens

\footnotetext{
${ }^{*}$ Correspondence: Dr. C. Giroud

Laboratoire de toxicologie analytique Institut Universitaire de Médecine Légale

Rue du Bugnon 21

$\mathrm{CH}-1005$ Lausanne

Tel.: +412131470 86

Fax: + 41213147090

E-Mail: christian.giroud@inst.hospvd.ch
}

of Cannabis sativa L. usually contain cannabinoids in the form of their carboxylic acid derivatives (Scheme) [3]. These tetrahydrocannabinolic acids, named $\Delta^{9}$-THC acid A and $\Delta^{9}$-THC acid B, readily decarboxylate into neutral $\Delta^{9}$-THC when heated and consequently cannot be examined directly by gas chromatography. In summer, the temperature of the plant parts is very likely high enough (foliar temperatures of $50-55{ }^{\circ} \mathrm{C}$ are quite common) to allow a partial decarboxylation of some acid cannabinoids. However, most of the neutral cannabinoids are probably formed slowly during the drying and the collection of the resinous fraction of the plant. Likewise, cannabis smoking results in the almost complete conversion of the acids into their decarboxylated active counterpart, i.e. $\Delta^{9}$-THC. Foodstuffs or beverages containing cannabis or cannabis extracts cooked or heated before administration (e.g. space cake, hemp Swiss fondue, hemp milk decoction) contain only trace amounts of the acids. The potency of a preparation is therefore better described by its total $\Delta^{9}$-THC concentration, usually as the $\%$ total $\Delta^{9}$-THC (i.e. neutral $\Delta^{9}-\mathrm{THC}+$ its acid counterparts) per dry weight of material. The taxonomy of cannabis also relies on the relative proportions of cannabinoids concentrations presented as the sum of neutral compound and the corresponding carboxylic acids [4]. In the case of oral administration of unheated plant material, the neutral fraction of $\Delta^{9}$-THC which is thought to be responsible of the psychoactive effects must be determined alone.

\section{Biosynthesis and Function of Cannabinoids}

The biosynthetic pathway of $\Delta^{9}$-THC has been delineated recently: condensation of geranyl pyrophosphate with olivetol carboxylic acid by a hemp transferase yields cannabigerolic acid [5] which in turn is a substrate for $\Delta^{9}$-THC acid A synthase [6]. In contrast to what was previously postulated, cannabidiol is not a precursor of $\Delta^{9}$-THC. Cannabidiol is very likely also formed as its acid derivative, i.e. cannabidiolic acid [7]. Although nonpsychoactive, this compound has been shown to possess anticonvulsant and anxiolytic properties. As far as we know, the regulation of $\Delta^{9}$-THC acid $\mathrm{A}$ synthesis and its interdependence with other pathways, e.g. the terpenoid pathway, are not yet elucidated and require further studies.

Several hypotheses have been put forward for the biological function of cannabinoids; most of them deal with an adaptation to environmental stress. A relation was suggested with drought and heat tolerance. Moreover, cannabinoids could provide protection against UV radiation, pathogens (antibiotic effect) and predators, e.g. aphids which might become ensnared in the resinous cannabinoids. 


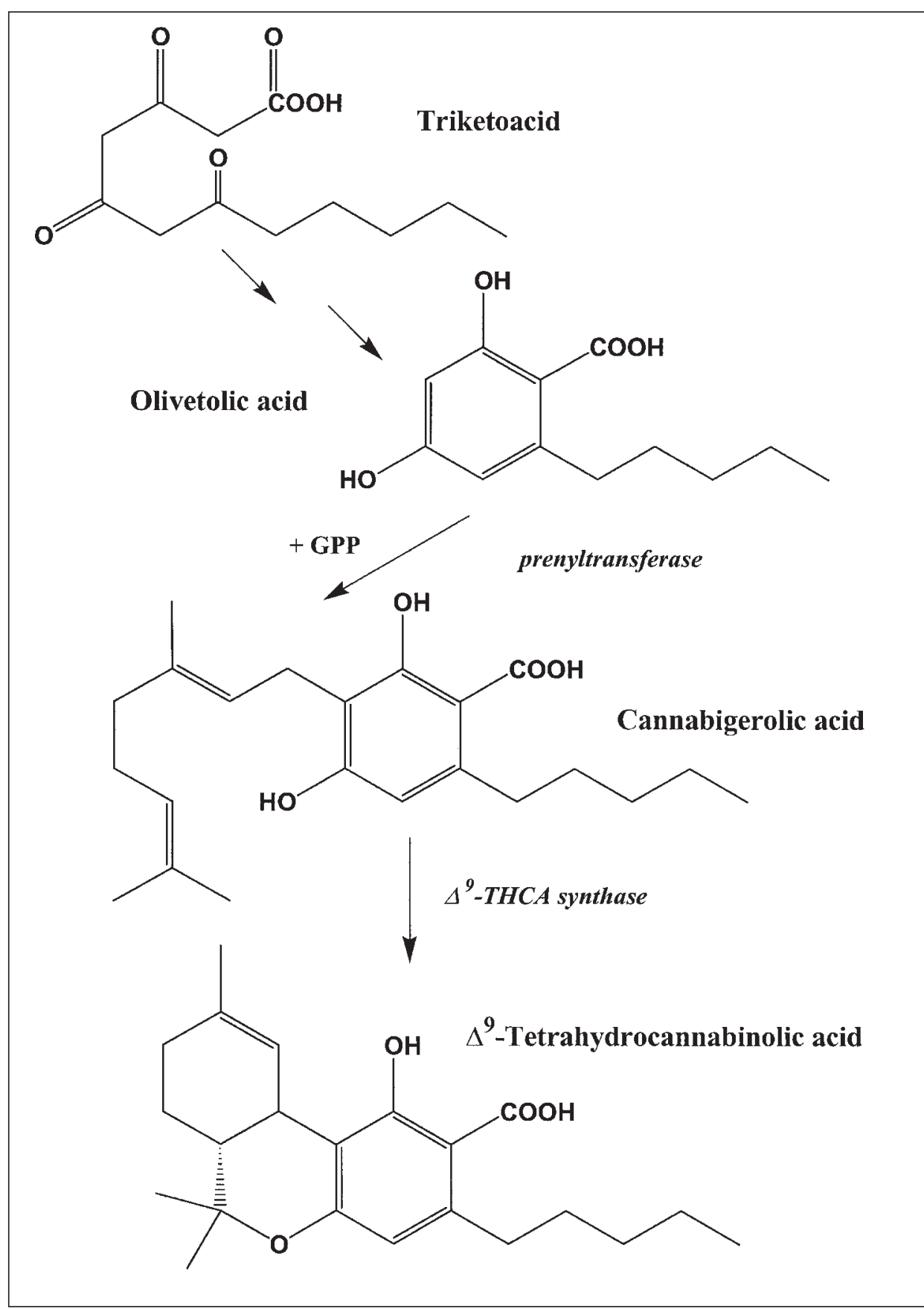

\section{The Hemp Plant}

Cannabis sativa L. grows outdoors as an annual, dioecious herb, i.e. it comprises both staminate and pistillate plants. Some varieties, however are monoecious, with both female and male flowers on the same plant. The plant is widely distributed throughout temperate and tropical zones. The hemp plant is characterized by palmate leaves with a toothed margin, usually composed of five to nine narrow lanceolate leaflets. The male flowers are gathered in panicles while female flowers form compact cymes. Female flowers have five perianth segments and are surrounded by large, persistent bracts. The abaxial surface of these bracts is densely covered with secretory and cystolith hairs. Short days initiate flowering and plants are photoperiodically adapted to terminate growth in relation to the length of their local growing season. There is no general agreement on the infrageneric taxonomic treatment of cannabis. Together with hops (Humulus lupulus L.), the hemp plant belongs to the Cannabaceae family which is member of the order of the Rosales. The leaf morphology was used to discriminate $C$. sativa L., $C$. indica Lam and $C$. ruderalis Janischevsky [8]. Since there appear to be no barriers to successful hybridization within the genus, it seems obvious to consider hemp as monotypic with one polymorphic species: Cannabis sativa $\mathrm{L}$
Scheme. Biosynthesis of $\Delta^{9}$-tetrahydrocannabinolic acid from triketoacid. The enzyme names are printed in italics.

[4]. Variation in the genus Cannabis is due in large part to selection by man. Two phenomena make easier the hybridization and intermixing of hemp plants: the escape of cultivated plants to the wild and their wind-pollination. The cannabinoid content is used to characterize the phenotypes or chemotypes belonging either to the 'drug' type or to the 'fiber' hemp type.

\section{Variation in $\Delta^{9}$-THC Content versus Age, Sex, and Plant Part}

The $\Delta^{9}$-THC is concentrated into a resin which is secreted into trichomes found on the small leaves (bracts) and bracteoles (leaf-like structure which encloses the ovary) of the flowering tops of the female plant [9]. The male plant produces an equivalent amount of active constituent which is found throughout the plant. The amount of resin found in the pistillate flowering tops is influenced by the climatic conditions of the plant, warm climates stimulate the synthesis of cannabinoids while cold weather lead to lesser amounts. The female hemp flowers remain fertile during a short time period. Hemp is known to build large amounts of new flowers as long as it does not become pollinated. Prevention of pollination stimulates the formation of new flowers and increases the yield of cannabinoids. This strategy is known by growers of drug cultivars as the 'sinsemilla' technique.

GC-FID analysis of the main parts of the hemp plant showed that the parts decrease in $\Delta^{9}$-THC content in the following order: bracts, flowers, leaves, smaller stems, roots, and seed. All plant parts contain cannabinoids [10][11]. Young floral leaves, those within the first nodes of the shoot apex, were found to contain 
higher concentrations of cannabinoids than older vegetative leaves [11]. Pollen grains and seeds were shown to contain low levels of cannabinoids. When examined by scanning electron microscopy, pollen grain samples were found to contain epidermal glandular trichomes (heads) intermixed with the pollen grains. The existence of gland heads, known to contain cannabinoids, suggests that the low level of cannabinoids represent a contaminant derived from glands rather than from pollen grains [11]. The presence of most of the cannabinoids in seeds could be also explained by external contamination. Indeed, the location of $\Delta^{9}$-THC within the achenes was found to be mainly on the outside surface of the seed coat, possibly the result of physical interaction with the plant bracts during processing [12]. Since manicured samples most likely contain different ratio of plant organs and tissues, cannabinoids values therefore represent a mean estimate of the total cannabinoid profile within the flowering portion of the plant. Until recently it was widely thought that the intoxicant properties of the resin of Cannabis were extremely subject to environmental modification. It now appears, however, that the intoxicant characteristics of the resin are reasonably conservative [4]. Moreover, no significant fluctuation in the relative amounts of the main cannabinoids $\Delta^{9}$-THC, cannabidiol and cannabichromene could be observed in the various types of Cannabis sativa analyzed so far [13].

\section{Criteria Aimed at the Distinction Between Fiber Hemp and Cannabis Belonging to Drug Type}

Studies and analysis of cannabis plants have indicated that cannabis strains or cultivars can be classified into two main chemical phenotypes according to their cannabinoid content. Fiber hemp strains or varieties generally contain more cannabidiol than $\Delta^{9}$-THC while resinous cannabis plants belonging to the 'drug type' are characterized by a weight ratio of $\Delta^{9}$-THC to cannabidiol higher than 1 . According to [14], hemp could be classified as given in the Table.

Taking into account the possible degradation of $\Delta^{9}$-THC into cannabinol, hemp has been classified into two major phenotypes according to the value of the cannabinoid ratio: $\left(\left[\Delta^{9}-\mathrm{THC}\right]+[\mathrm{CBN}]\right) /$ [CBD]. If the ratio exceeded 1 , hemp plants were classified as 'drug phenotype', otherwise as belonging to the 'fiber phenotype' [15].

\section{The Current Swiss Situation: Legislation}

The cultivation of hemp is prohibited in many countries, except for $\Delta^{9}$-THCpoor cultivars. In Switzerland, the cultivation of hemp is not prohibited provided it is not done for the purpose of producing narcotic drugs [16][17]. Anybody growing hemp with a $\Delta^{9}$-THC content higher than $0.3 \%$ is however liable to investigation and prosecution. Since the decision of the Swiss Federal Supreme Court of 29 August 1991, Cannabis, even in large quantities 'cannot endanger the health of many individuals'. Subsidies are provided for the cultivation of $\Delta^{9}$-THC-poor strains of Cannabis by the Federal Office of Agriculture to develop new sources of renewable materials. A list of saleable varieties of hemp seeds was drawn up in 1998 [18]. The list which encompasses about a dozen varieties, e.g. Fedora 19, Félina 34, Kompolti, Uniko-B is periodically brought up-to-date. All these strains are characterized by a $\Delta^{9}$-THC level lower than $0.3 \%$. Regulations concerning the marketing of foods and beverages containing hemp products were issued by the Swiss Federal Office of Public Health in 1998 [19]. On the whole, the limit was established between 0.00002 and $0.005 \%$ $\Delta^{9}$-THC. For instance, alcoholic beverages containing more than $0.2 \mathrm{mg} / \mathrm{kg}$ no longer constitute foodstuffs within the meaning of the Swiss Food Act. A recent settlement issued by the Swiss Federal Supreme Court established that the afore-

Table. Classification of hemp chemotypes into fiber and resinous Cannabis according to [14]

\begin{tabular}{llll} 
Phenotype & $\Delta^{9}-\mathrm{THC}[\%]$ & CBD [\%] & $\Delta^{9}-\mathrm{THC} / \mathrm{CBD}$ \\
\hline Fiber hemp & $<0.5$ & $>0.5$ & $<1$ \\
\hline Resinous Cannabis & $>0.5$ & $<0.5$ & $>1$
\end{tabular}

mentioned levels can be used to determine whether a product must be considered as a narcotic or not [20].

Hemp has become a dual-use crop, i.e. it may be both taken as an illicit narcotic drug (e.g. marijuana) and used legally in agriculture, for instance, for the production of fibers, foodstuffs, cosmetics. Some materials can be used either as a drug or as a legal article, typically, hemp coins might be gathered by collectors fond of numismatics or used as a recreational or narcotic drug [21].

\section{Cannabis Products}

For drug purposes, either the resin (hashish) is used or the flowering tops of the female plant (marijuana). The percentage of resin in hashish determines its color, consistency and $\Delta^{9}$-THC content. Hashish oil is an extraction residue obtained after treatment of dried hemp tips with an organic solvent (e.g. 2-propanol). Hashish can be obtained from pollen, also called in Holland pollem or polm. One should not mistake 'pollen' for the male reproductive parts of the hemp plant. 'Pollen' is extracted and sieved mechanically with a pollinator from the blooming female plant after harvesting and contains therefore no more than traces of pollen!

\section{Sampling of Cannabis Material}

According to recent guidelines issued by the group of Forensic Chemistry of the Swiss Society of Forensic Medicine, Cannabis sampling must performed as follows:

\section{a. Fields}

Thirty flowering tops from 30 different female plants are taken along the diagonal of the field. No sampling must be performed on the sides of the plantation. The best time to collect the specimens is just before harvesting, in the afternoon of a dry day. The cannabinoid content of a mixed sample of 30 plants is considered to be a reliable approximation of the average content of the field. When possible, it is recommended to let the farmer harvest the crop and to take dried samples from it for analysis. Formerly, the European Community procedure to determine total $\Delta^{9}$-THC content in fiber hemp cultivars dictated a sample size of 500 individual plants to be analyzed as a mixed sample [22]. The cutoff value was $0.3 \%$ total $\Delta^{9}$-THC in the dry matter. In 
2000, new guidelines were issued by the European Union. The size of the sample was decreased from 500 plants to 50 and the value of the cutoff lowered to $0.2 \%$ [23]. Investigations carried out by [24] indicated that a mixed sample of 20 plants could be considered to be a reliable approximation of the mean content of a small plot of plants.

\section{b. Raw Material}

Marijuana samples are directly blended with a mixer and the homogenous powder analyzed as described below. Two samples of at least $1 \mathrm{~g}$ each are taken per sheet of hashish with a metal punch. Sampling near the sides must be avoided. The samples are then pulverized with a mixer.

\section{c. Foodstuffs and Beverages}

These samples can be prepared as described in ref. [27].

\section{Analysis of Cannabinoids in Hemp Plants and Derivatives}

Capillary gas-chromatography with flame ionization detection (GC-FID) and mass spectrometry (GC-MS) and highperformance liquid chromatography with UV (HPLC-UV) or diode array detection (HPLC-DAD) are generally used to establish the chemical profile of Cannabis samples and to carry out cannabinoid determinations. Without derivatization, GC methods yield the so-called 'total$\Delta^{9}$-THC' concentration that corresponds to the total $\Delta^{9}$-THC (i.e. neutral $\Delta^{9}$-THC + its acid counterparts). Formation of the silylated or alkylboronate derivatives stabilize the acids and allow the separate determinations of $\Delta^{9}$-THC and of its acid counterparts ( $\Delta^{9}$-THC-A and -B) [25][26]. For food control purposes, HPLC is generally preferred over GC techniques. A solvent-programmed reversed-phase HPLC method with UV and fluorescence detection for the determination of $\Delta^{9}$-THC-A and $\Delta^{9}$-THC in foods such as edible oil, herb-teas, herbal hemp or hempseed has been presented recently [27]. The method was adapted from [28]. One main drawback of these methods is that no certified reference standard of $\Delta^{9}$-THC-A is commercially available, $\Delta^{9}$-THC-A has therefore to be isolated, purified and identified before using it as a standard for quantification.

Before analysis by known chromatographic procedures, cannabinoids must be extracted from plant tissues or from foodstuffs. First, plant tissues are generally dried overnight in an oven, then manicured, pulverized with a mixer (e.g.
Retsch Grindomix) and sieved to yield a homogenous and fine powder. A small aliquot of the powder is extracted with an organic solvent (e.g. petrol ether, methanol/dichloromethane $9: 1(\mathrm{v} / \mathrm{v}))$ with or without sonication overnight or during a few minutes only. According to [29], some hashish and hash oil samples have exhibited the unusual property of being insoluble in organic solvents, but soluble in water. In order to dissolve them, they must be prepared by partitioning small aliquots between chloroform and water. All analyses must be performed in triplicate. Squalane, 4-androstene-3,17-dione, tetracosane can be used as an internal standard for cannabinoids determinations. $\Delta^{9}-\mathrm{THC}$ or cannabinol, which is more stable, can be used as standards to set up calibration curves (Recommendations for the analysis of hemp, Group of Forensic Chemistry, Swiss Society of Forensic Medicine, 2001).

\section{Other Strategies Used for the Classification of Hemp Plants}

Many attempts have been made to characterize hemp cultivars of different origin. One strategy was to use electrophoretic profiles of seed proteins [30]. Various DNA 'profiling' techniques have been shown to assess the genetic relatedness of species, varieties, cultivars and even individuals [31][32]. For instance, RAPD (Random Amplified Polymorphic DNA using the Polymerase Chain Reaction) was used to obtain informative and reproducible fragments, that showed clear differences between samples from different sources [32]

Received: January 29, 2002

[1] R. Mechoulam, A. Shani, H. Edery, Y. Grunfeld, Science 1970, 169, 611.

[2] S. Agurell, S. Levander, M. Binder, A. Bader-Bartfai, B. Gustafsson, K. Leander, J.-E. Lindgren, A. Ohlsson, B. Tobisson, in 'The pharmacology of marihuana', Eds. M.C. Braude, S. Szara, Raven Press, New York, 1976.

[3] R. Mechoulam, Science 1970, 168, 1159.

[4] E. Small, A. Cronquist, Taxon 1976, 25, 405.

[5] M. Fellermeier, M.H. Zenk, FEBS Letters 1998, 427, 283.
[6] F. Taura, S. Morimoto, Y. Shoyama, R. Mechoulam, J. Am. Chem. Soc. 1995, 117, 9766.

[7] R. Mechoulam, S. Ben-Shabat, Nat. Prod. Rep. 1999, 16, 131.

[8] L.C. Anderson, Harvard University Botanical Museum leaflets 1980, 28, 61.

[9] E.S. Kim, P.G. Mahlberg, American J. Botany 1997, 84, 336.

[10] P.S. Fetterman, E.S. Keith, C.W. Waller, O. Guerrero, N.J. Doorenbos, M.W. Quimby, J. Pharmac. Sciences 1971, 60, 1246.

[11] J.K. Hemphill, J.C. Turner, P.G. Mahlberg, J. Nat. Prod. 1980, 43, 112.

[12] S.A. Ross, Z. Mehmedic, T.P. Murphy, M.A. ElSohly, J. Anal. Toxicol. 2000, 24, 715.

[13] I . Barni-Comparini, S. Ferri, F. Centini, For. Sci. Int. 1984, 24, 37.

[14] G. Fournier, M.R. Paris, Plant Med. Phytother. 1979, 13, 116.

[15] P.S. Fetterman, E.S. Keith, C.W. Waller, O. Guerrero, N.J. Doorenbos, M.W Quimby, J. Pharmaceut. Sciences 1971 60, 1246.

[16] C. Giroud, L. Rivier, TIAFT Bulletin 1996, 26, 30 .

[17] C. Giroud, A. Broillet, M. Augsburger, W. Bernhard, L. Rivier, P. Mangin, Praxis 1999, 88, 113 .

[18] Ordinance SR 916.151.6 of the Swiss Federal Office of Agriculture issued December 7, 1998.

[19] Ordinance SR 817.021.23 of the Swiss Federal Office of Public Health on Foreign substances and ingredients in food products on January 30, 1998.

[20] Swiss Federal Supreme Court of Appeal, judgment of the March 13, 2000.

[21] C. Giroud, A. Broillet, M. Augsburger, W. Bernhard, L. Rivier, P. Mangin, Toxicorama 1998, 11, 51.

[22] G. Fournier, O. Beherec, S. Bertucelli, J.P. Mathieu, Annales de Toxicologie Analytique 2001, 13, 275.

[23] Règlement (CE) n²860/2000 de la Commission du 27 décembre 2000. Journal Officiel des Communautés européennes 28/12/2000.

[24] E.P.M. de Meijer, H.J. van der Kamp, F.A. van Eeuwijk, Euphytica 1992, 62, 187.

[25] S. Billets, F. El-Feraly, P.S. Fetterman, C.E. Turner, Org. Mass Spectrom. 1976 11,741 .

[26] D.J. Harvey. Biomed, Mass Spectrom. 1977, 4, 88

[27] O. Zoller, P. Rhyn, B. Zimmerli, J. Chromatogr. A 2000, 872, 101.

[28] T. Lehmann, R. Brenneisen, J. Liquid Chromatogr. 1995, 18, 689.

[29] M.A. ElSohly, S.A. Ross, Z.Mehmedic, R. Arafat, B. Yi, B. Banahan, J. Forensic Sci. 2000, 45, 24.

[30] C. Lucchese, G. Venturi, M.T. Amaducci, A. Lovato, Seed Sci. \& Technol. 2001, 29, 239.

[31] R.G. Cole, A. Linacre, J.W. Thorpe, N.D. Watson, Sciences \& Justice 1995, 35, 169.

[32] V. Jagadish, J. Robertson, A. Gibbs, For. Sci. Int. 1996, 79, 113. 